\title{
INFLUÊNCIAS HISTÓRICAS NA ABORDAGEM DE LIVROS DIDÁTICOS DE MATEMÁTICA
}

\author{
Alan Raniel Borges da Cruz ${ }^{1}$
}

\begin{abstract}
RESUMO
O presente artigo objetiva levar o leitor a uma reflexão da prática docente na área de matemática. Para isso, foram buscadas informações históricas sobre a década de 1970 até o presente, tanto em artigos e obras referentes ao período, como analisando livros didáticos de matemática referentes ao espaço de tempo. No total, seis livros didáticos, variando o ano de publicação entre 1971 e 2012. Foram consultados autores como Burigo (1990), D'Ambrósio (2008), DobrovolskI (2009), Iskandar (2002), Kline (1976), Mathias (2012) e Valente (2007), para entender melhor cada momento histórico da matemática no Brasil, tendo o período indicado como referência. Os Parâmetros Curriculares Nacionais (PCNs) de matemática também foram fundamentais para justificar a relação forte entre uma ciência e história. Espera-se que ao final da leitura esteja clara a relação da matemática com sua história, bem como a história do país e ainda, que a educação matemática é dinâmica e acompanha o momento social do país.
\end{abstract}

Palavras Chave: Livro didático. História da matemática. Abordagens contextualizadas.

\section{INTRODUÇÃO}

O objetivo do presente artigo não é apontar uma maneira correta ou errônea de trabalho ou abordagem. E, sim, levar a uma reflexão da prática docente na área de matemática ao explicitar as influências históricas que aconteceram da década de 1970 até o presente, tendo como base livros didáticos de matemática. Conforme Brasil (1997), uma ciência não é atemporal, nem descontextualizada com a história, de modo que, conhecer bem os processos pelos quais a mesma passou na formação para a conjectura atual é uma forma de aprofundar também na própria ciência.

Será feito a princípio um histórico resumido da história do Brasil no contexto da matemática, objetivando a contextualização histórica com a disciplina. Em seguida, tem-se a apresentação das formas de abordagens de conteúdos matemáticos em seis livros didáticos de matemática brasileiros, com publicações em anos distintos.

\footnotetext{
${ }^{1}$ Licenciado em Matemática pela Pontifícia Universidade Católica de Minas Gerais (PUC-MINAS) e, atualmente, é professor de Matemática na Prefeitura Municipal de Betim e professor de Física em preparatório para o ENEM no IMEC. (http://lattes.cnpq.br/0543062753314902). E-mail: alan_raniel@yahoo.com.br.
} 


\section{BREVE HISTÓRICO DA MATEMÁTICA NO BRASIL}

A Proclamação da República, datada em 15 de novembro de 1889, será marcada como o ponto de partida nesse trabalho, porém ressaltando que os acontecimentos anteriores não são menos importantes. Inicia-se junto da república um período de certa estagnação matemática e científica no Brasil. Existe uma relação desse fato com as influências do movimento positivista de Auguste Comte (1789-1857). O positivismo teve grande influência na construção da república brasileira e como já era o modo de pensar utilizado nas faculdades, conseguiu consolidar-se no âmbito de toda matemática nacional (D’AMBROSIO, 2008).

Para Comte, seu movimento poderia ser uma forma de garantir a organização da sociedade de forma racional, era um modo de pensar, bem como de promover transformações sociais. Privilegiava o que era "prático, útil, objetivo, direto e claro" (ISKANDAR; LEAL, 2002, p. 3).

O positivismo influenciou não somente a construção da república do Brasil, mas o modo como se estuda e ensina desde o século XIX até os dias atuais, basta reparar no que está escrito na bandeira nacional brasileira (“Ordem e Progresso"), é uma frase intimamente ligada ao positivismo. E a distribuição do currículo escolar com disciplinas bem divididas e fragmentas é uma influência do movimento. Lembrando ainda que era feita restrição de interação entre as diferentes disciplinas e que as ciências exatas eram exaltadas em detrimento das humanas (ISKANDAR; LEAL, 2002).

Os livros didáticos, no caso da matemática, escritos aproximadamente na metade do século XX, eram compostos por muitas definições. As operações eram apresentadas com muitas regras de como resolvê-las e as justificativas para resolução focadas apenas em teorias matemáticas puras (D’AMBROSIO, 2008).

Também se destaca na história da matemática no Brasil o Movimento da Matemática Moderna (MMM), iniciando-se na década de 1960. Conforme Búrigo (1990) o movimento buscava atualização e adequação do ensino de matemática para condizer com a sociedade que estava em progresso técnico, dava ênfase para métodos de cálculos sem qualquer contexto que não seja matemática pura e os exercícios de fixação eram formais e voltados apenas para conteúdos matemáticos com grande ênfase em demonstrações e deduções de conceitos.

Interligando as ideias de Pinto e Ferreira (2006) com Valente (2007), Dobrolski e Pinto (2009) e também Mathias (2012), os avanços tecnológicos, na década de 1950, em todo o mundo refletiram em preocupações para o governo, não só brasileiro, quanto à formação 
científica que a população possuía, em seguida surgiu uma ideia de reforma para o ensino de matemática. Então, foi fundado o Grupo de Estudo do Ensino da Matemática (GEEM) em 1961, com propostas de matemática rígida, menos instrumental e pouco prática. O GEEM também objetivava a divulgação do MMM.

Durante o período de 1970 até 1990, a maioria dos livros de matemática publicados no Brasil foi influenciada pelo MMM, nos mesmos, não existia contextualização da matemática. A apresentação da matemática era feita de forma tão simbólica e algébrica que chegou ao ponto de acontecer uma separação entre os livros de álgebra e geometria, como se fossem duas disciplinas diferentes. A álgebra, contudo, foi privilegiada e era denominada matemática, a outra, geometria. Algumas escolas passaram a contratar professores diferentes, a matemática então estava descontextualizada até mesmo da geometria, como se fosse outra disciplina, e não apenas matemática como todo (MATHIAS, 2012).

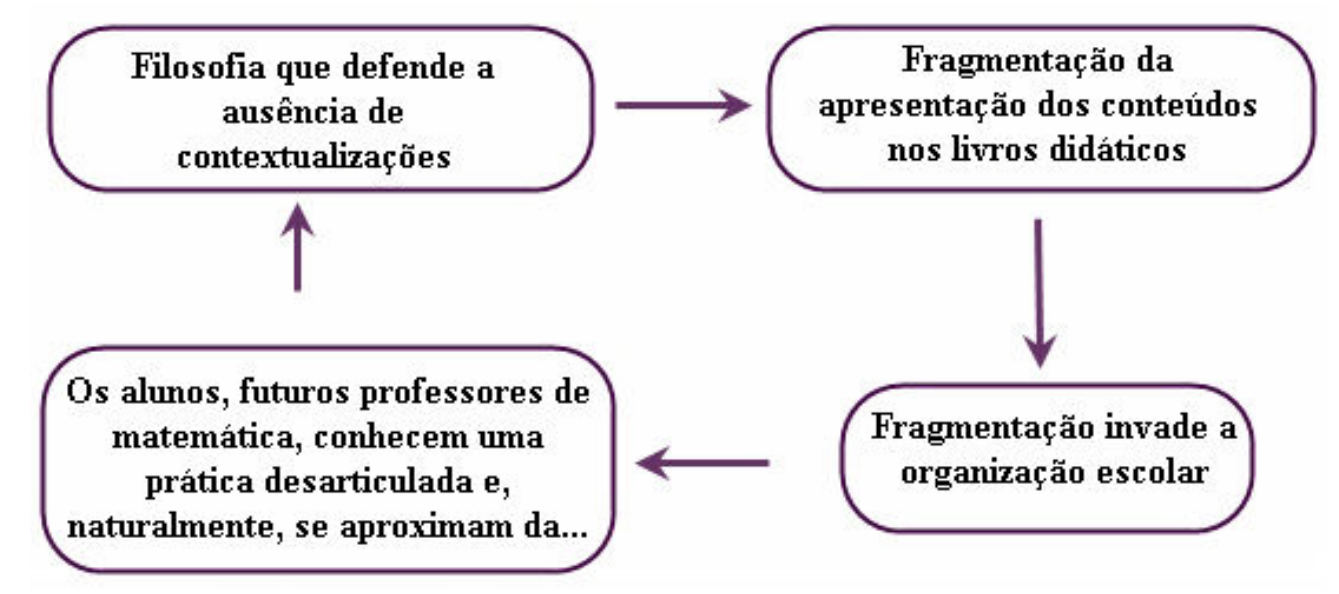

Figura 1 - Implicações da fragmentação matemática Fonte: Mathias (2012).

$\mathrm{Na}$ Matemática Moderna, a abordagem dos livros tinha uma proposta de inovar, deixando de lado a memorização de processos, propondo que o ensino da matemática deveria acontecer por uma lógica capaz de dar entendimento ao raciocínio que existia oculto no método. A abordagem lógica proposta iniciava-se em axiomas e postulados, provavam-se dedutivamente as proposições e por fim, tinham-se conclusões. A geometria perdeu seu caráter ilustrativo e era ensinada assim, com provas de deduções. (KLINE, 1976).

A situação tornava-se então cíclica durante o período de influência do MMM, conforme o esquema apresentado por Mathias (2012, p. 77).

Somente na década de 90 foi possível verificar ações contrárias à fragmentação da matemática nos livros didáticos, porém, apenas para o ensino fundamental, o ensino médio ForSci.: r. cient. IFMG, Formiga, v. 4, n. 1, p. 102-113, jan./jun. 2016. 
ainda permaneceu com um padrão rígido e pautado em fórmulas e acesso rápido de informações para uso em vestibulares (MATHIAS, 2012).

\section{METODOLOGIA PARA ANÁLISE DOS LIVROS}

\subsection{Análise 1}

Para iniciar a análise, segue um quadro atribuindo uma "identificação" para cada livro. Dando prosseguimento foi construída uma comparação com os conteúdos propostos por cada livro para o que atualmente é $9^{\circ}$ ano. Para construir a tabela foram anotados todos os conteúdos que aparecem em todos os livros selecionados. Vale lembrar que conforme a época história tomada como referência, poderia ser conhecida como $8^{\mathrm{a}}$ série, ou ainda, $4^{\mathrm{o}}$ ano do ciclo ginasial. No total foram analisadas 7 obras de anos distintos, desde 1971 até uma recente de 2012.

\begin{tabular}{|c|c|}
\hline Obra & $\begin{array}{l}\text { Denominação para } \\
\text { o artigo }\end{array}$ \\
\hline $\begin{array}{l}\text { MORANDI, H. Matemática: método moderno. Curso médio: ciclo ginasial. } \\
\text { Rio de Janeiro: Paulo de Azevedo,1971. p. } 325 .\end{array}$ & A \\
\hline SANGIORGI, O. Matemática. 8ª série. São Paulo: Nacional, 1978. p. 198. & B \\
\hline $\begin{array}{l}\text { GIOVANNI, J. R.; CASTRUCCI, B. A conquista da matemática: teoria, } \\
\text { aplicação, } 8^{\text {a }} \text { série. São Paulo: FTD, 1985. } 192 \text { p. }\end{array}$ & $\mathrm{C}$ \\
\hline $\begin{array}{l}\text { GIOVANNI, J. R.; GIOVANNI JÚNIOR, J. R. Matemática: pensar e } \\
\text { descobrir, 8. São Paulo: FTD, 1996. } 303 \text { p. (Coleção pensar e descobrir). }\end{array}$ & $\mathrm{D}$ \\
\hline $\begin{array}{l}\text { MATSUBARA, R.; ZANIRATTO, A. A. Matemática: história, evolução, } \\
\text { conscientização, } 8 \text { a série. 2. ed. São Paulo: IBEP, 2002. } 215 \text { p. (Coleção Big } \\
\text { Mat). }\end{array}$ & $\mathrm{E}$ \\
\hline $\begin{array}{l}\text { MORI, I.; ONAGA, D. S. Matemática: ideias e desafios, } 9^{\circ} \text { ano. 17. ed. São } \\
\text { Paulo: Saraiva, 2012. } 320 \text { p. }\end{array}$ & $\mathrm{F}$ \\
\hline
\end{tabular}

Quadro 1 - Lista de obras analisadas

Fonte: Raniel (2015).

\begin{tabular}{|l|c|c|c|c|c|c|}
\cline { 2 - 8 } \multicolumn{1}{c|}{} & \multicolumn{7}{c|}{ Presença na obra } \\
\hline Conteúdo & A & B & C & D & E & F \\
\hline Circunferência e círculo & $\mathrm{X}$ & $\mathrm{X}$ & $\mathrm{X}$ & $\mathrm{X}$ & & $\mathrm{X}$ \\
\hline Conjunto dos números inteiros & $\mathrm{X}$ & & & & $\mathrm{X}$ & \\
\hline Conjunto dos números irracionais & $\mathrm{X}$ & $\mathrm{X}$ & & & $\mathrm{X}$ & \\
\hline Conjunto dos números naturais & $\mathrm{X}$ & & & & $\mathrm{X}$ & \\
\hline Conjunto dos números reais & $\mathrm{X}$ & & & & $\mathrm{X}$ & \\
\hline Conjunto dos números imaginários & $\mathrm{X}$ & & & & & \\
\hline Estatística Básica, tratamento da informação & & & & $\mathrm{X}$ & & $\mathrm{X}$ \\
\hline Equações biquadradas & $\mathrm{X}$ & & $\mathrm{X}$ & $\mathrm{X}$ & $\mathrm{X}$ & $\mathrm{X}$ \\
\hline Equações do 2 $2^{\circ}$ grau & & $\mathrm{X}$ & $\mathrm{X}$ & $\mathrm{X}$ & $\mathrm{X}$ & $\mathrm{X}$ \\
\hline
\end{tabular}




\begin{tabular}{|l|c|c|c|c|c|c|}
\hline Equações irracionais & $\mathrm{X}$ & & & $\mathrm{X}$ & $\mathrm{X}$ & $\mathrm{X}$ \\
\hline Função - Noções iniciais & $\mathrm{X}$ & $\mathrm{X}$ & $\mathrm{X}$ & $\mathrm{X}$ & $\mathrm{X}$ & $\mathrm{X}$ \\
\hline Função do $1^{\circ}$ grau & $\mathrm{X}$ & $\mathrm{X}$ & $\mathrm{X}$ & $\mathrm{X}$ & $\mathrm{X}$ & $\mathrm{X}$ \\
\hline Função do $2^{\mathrm{o}}$ grau & $\mathrm{X}$ & $\mathrm{X}$ & $\mathrm{X}$ & $\mathrm{X}$ & $\mathrm{X}$ & $\mathrm{X}$ \\
\hline Juros simples e composto & & & & & $\mathrm{X}$ & \\
\hline Medidas de área & $\mathrm{X}$ & $\mathrm{X}$ & $\mathrm{X}$ & $\mathrm{X}$ & $\mathrm{X}$ & $\mathrm{X}$ \\
\hline Medidas de comprimento & $\mathrm{X}$ & $\mathrm{X}$ & $\mathrm{X}$ & $\mathrm{X}$ & $\mathrm{X}$ & $\mathrm{X}$ \\
\hline Medidas de volume & & & & & $\mathrm{X}$ & \\
\hline $\begin{array}{l}\text { Polígonos convexos circunscritos a uma } \\
\text { circunferência }\end{array}$ & & & & $\mathrm{X}$ & \\
\hline $\begin{array}{l}\text { Polígonos regulares e inscritos numa } \\
\text { circunferência }\end{array}$ & $\mathrm{X}$ & $\mathrm{X}$ & $\mathrm{X}$ & $\mathrm{X}$ & $\mathrm{X}$ & $\mathrm{X}$ \\
\hline Porcentagem & & & & & $\mathrm{X}$ & \\
\hline Potências e Radicais & $\mathrm{X}$ & $\mathrm{X}$ & $\mathrm{X}$ & $\mathrm{X}$ & $\mathrm{X}$ & $\mathrm{X}$ \\
\hline Proporção e razão, teorema de Tales & $\mathrm{X}$ & $\mathrm{X}$ & $\mathrm{X}$ & $\mathrm{X}$ & $\mathrm{X}$ & $\mathrm{X}$ \\
\hline Regra de 3 simples e composta & & & & & $\mathrm{X}$ & \\
\hline $\begin{array}{l}\text { Relações métricas no triângulo retângulo, } \\
\text { Teorema de Pitágoras }\end{array}$ & $\mathrm{X}$ & $\mathrm{X}$ & $\mathrm{X}$ & $\mathrm{X}$ & $\mathrm{X}$ & $\mathrm{X}$ \\
\hline Relações de um triângulo qualquer & $\mathrm{X}$ & $\mathrm{X}$ & $\mathrm{X}$ & $\mathrm{X}$ & & \\
\hline $\begin{array}{l}\text { Relações trigonométricas no triângulo } \\
\text { retângulo }\end{array}$ & $\mathrm{X}$ & $\mathrm{X}$ & $\mathrm{X}$ & $\mathrm{X}$ & $\mathrm{X}$ & $\mathrm{X}$ \\
\hline Semelhança de figuras planas & $\mathrm{X}$ & $\mathrm{X}$ & $\mathrm{X}$ & $\mathrm{X}$ & $\mathrm{X}$ & $\mathrm{X}$ \\
\hline Semelhança de triângulos & $\mathrm{X}$ & $\mathrm{X}$ & $\mathrm{X}$ & $\mathrm{X}$ & $\mathrm{X}$ & $\mathrm{X}$ \\
\hline Sistema de equações & $\mathrm{X}$ & & & & $\mathrm{X}$ & $\mathrm{X}$ \\
\hline Similitude geral ou homotetia & & $\mathrm{X}$ & & & & \\
\hline
\end{tabular}

Quadro 2 - Presença de conteúdos nas obras analisadas Fonte: Raniel (2015).

Com o Quadro 2 é possível visualizar que não houve grandes mudanças nos conteúdos apresentados para o atual $9^{\circ}$ ano, desde 1971 até os dias atuais. O que difere muito ao longo do tempo quando comparamos os livros é a forma como os conteúdos são abordados. Conforme Mathias (2012), alguns livros influenciados pelo MMM apresentavam divisão, um livro de álgebra e outro de geometria, para a mesma série. Os livros escolhidos para análise possuem ambos os conteúdos num único volume, sem separação.

As recomendações para o ensino de matemática no Brasil indicam eixos para o trabalho, tratando todos os eixos com igual importância e como parte de um todo denominado "matemática”. Logo, é pertinente recorrer à seguinte fala:

Há um razoável consenso no sentido de que os currículos de Matemática para o ensino fundamental devam contemplar o estudo dos números e das operações (no campo da Aritmética e da Álgebra), o estudo do espaço e das formas (no campo da Geometria) e o estudo das grandezas e das medidas (que permite interligações entre os campos da Aritmética, da Álgebra e da Geometria). (BRASIL, 1997, p 53).

ForSci.: r. cient. IFMG, Formiga, v. 4, n. 1, p. 102-113, jan./jun. 2016. 
Pensando no trabalho com os campos da matemática indicados por Brasil (1997), temse ainda um quarto campo, o do tratamento da informação, esse serve como ferramenta para a cidadania, uma vez que o cidadão necessita ter conhecimento sobre tantas informações que chegam para si e ainda, que o tratamento da informação consegue interagir com qualquer outro campo de alguma forma. No fim, todos os campos devem ser trabalhados de forma integrada e um contribuindo com o outro, uma vez que tudo é matemática, sem maior ou menor importância. Logo, a análise foi focada em forma de abordagem do conteúdo e não na forma como são divididos (BRASIL, 1997).

\subsection{Análise 2}

Foi escolhido o conteúdo de função, uma vez que é comum a todas as obras analisadas, em seguida foi feita uma comparação de como é abordado em cada uma. Reiterando que o objetivo do artigo não é criticar as obras, tendo em vista que cada uma tem seu momento histórico, contexto e justificativa, deseja-se explicitar a influência histórica na forma de abordagem de cada livro. Avaliando então desde a noção inicial até a definição das funções polinomiais de $1^{\mathrm{o}}$ grau.

\subsubsection{Livro A}

O conceito de função é apresentado de maneira muito formal, com direito a rigor matemática que atualmente seria empregado no ensino superior. Não somente isso, mas antes de definir função, são definidas as relações e correspondências, dando ênfase num rigor muito elevado para o uso de símbolos matemáticos, no produto cartesiano, diagramas, conjuntos imagem, domínio, contradomínio, dentre outros. Veja a formalização do conceito:

[...] quando certa lei define uma relação entre dois conjuntos, e a correspondência entre todos os elementos do primeiro conjunto para os elementos do segundo é unívoca, tal relação é uma aplicação, função ou transformação dos elementos do primeiro nos elementos do segundo.

Compreende-se então que, nas aplicações, o conjunto de partida é sempre igual ao domínio da relação: cada elemento do conjunto de partida tem um, e um só, correspondente ou imagem no conjunto B, logo, todo elemento de A é sujeito da relação e por isso pertence ao $\mathrm{D}(\mathrm{R})$. Isto não quero dizer que o conjunto de chegada deva se confundir necessariamente com o conjunto das imagens, pois pode ocorrer que algum elemento de B seja imagem (única) de mais de um sujeito, ou não seja imagem de nenhum. 
No grafo de uma aplicação devemos notar a condição fundamental: De todo elemento de A sai uma, e uma única, flecha para B (MORANDI, 1971, p. 32).

Para concluir a definição, é indicado um diagrama como o seguinte:

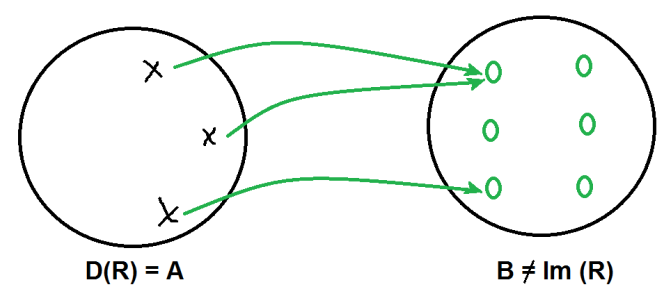

Figura 2 - Grafos retirados de Morandi (1971, p. 32)

Fonte: Morandi (1971).

\subsubsection{Livro B}

O capítulo sobre funções inicia-se falando que o conteúdo de relação já foi estudado e que é o momento de estudar uma relação binária especial, que é chamada de função. É feita uma breve retomada com o exemplo de "associar a cada criança o seu pai", com observações acerca de um diagrama similar ao apresentado pela obra anterior.

São feitos esclarecimentos sobre a relação “criança x pai”. Em seguida apresenta-se vários diagramas similares, alguns com a informação de serem uma função, outros de não ser. Por fim, é dada uma observação, como se fosse a definição de função.

Função: 1. Cada ponto é origem de uma única flecha.

2. Os pontos podem ser extremidade de mais de uma flecha ou nenhuma. (SANGIORGI, 1978, p. 47)

Daí por diante já são apresentados exercícios de aplicação de cálculos, após mais de 23 cálculos fala-se sobre a função estar presente no dia-a-dia, como na produção anual de carros no Brasil, ou a de temperatura e volume do mercúrio em um termômetro. Por fim, define-se domínio, imagem e notação de forma similar, apenas cálculos diretos e diagramas. Todos exercícios apresentados não possuem contexto, apenas cálculos diretos.

\subsubsection{Livro C}

Neste já aparecem referências sobre situações mais cotidianas e reais onde é possível fazer uso de funções. Tem-se o preço do álcool para abastecer um carro apresentado numa 
tabela, onde conforme o litro aumenta em uma unidade, o preço a pagar aumenta em 3,10, para por fim, prever qual seria o valor a pagar de 55 litros. Ao final do raciocínio tem-se uma conclusão:

a) O preço y a pagar é dado em função do número $\mathrm{x}$ de litros colocados no tanque.

b) A relação $y=3,10 x$ chama-se de fórmula matemática dessa função. (GIOVANNI; CASTRUCCI, 1985, p. 86).

Em seguida, problemas relacionando perímetro e área de um quadrado. Logo, a abordagem torna-se basicamente cálculos e representação em diagramas. A quantidade de exercícios em relação ao anterior é relativamente menor, porém, continuam exercícios sem contextualização com a vida prática, aplicações de cálculos prevalecem.

\subsubsection{Livro D}

As explicações sobre funções são iniciadas com muitos exemplos contextualizados. As situações indicam carros em movimento, relações de área de terrenos, problemas com notas de aluno, jogos de futebol, gráficos de impostos, dentre outros. O livro apresenta formas práticas de utilizar uma função para resolver problemas na vida real. O conteúdo está dividido metade com exemplos do que é uma função e outra metade propondo cálculos e diagramas. Nos exercícios verifica-se 11 de problemas com contexto em contrapartida a oito de resolução direta privilegiando cálculos. Após muitos exemplos e exercícios a função é definida como:

Sendo A e B dois conjuntos não-vazios, uma relação entre A e B é chamada função
quando a cada elemento x do conjunto A está associado um único elemento y do
conjunto B (GIOVANNI; GIOVANNI JÚNIOR, 1996, p. 110).

\subsubsection{Livro E}

A contextualização existe antes de uma definição "formal", aparecem situações sobre volume, massa, e outros, tal qual no livro D. A grande novidade é que já aparecem muitas imagens e em quatro páginas referentes ao início do conteúdo de funções já aparecem duas caixas de texto de uma seção do livro denominada "Você sabia". A primeira ao fazer referência ao teste de QI como uma função, fala do quanto o conceito é questionável, fazendo referência à revista Nova Escola da Editora Abril. E o segundo "Você sabia" descreve um 


\section{FORSCIENCE}

CRUZ, A. R. B. da. Influências históricas na abordagem de livros didáticos de matemática

serviço da UNICAMP a respeito de orientações sobre derramamento de óleo no oceano. Recomenda-se ainda uma pesquisa sobre profissões indicando geólogo, biólogo, engenheiro, oceanógrafo, tal pesquisa busca relacionar a importância da compreensão de funções no trabalho de tais profissões.

O conceito de função não é definido com linguagem exclusivamente matemática. Apenas quando se fala da função polinomial de $1^{\circ}$ grau é apresentada uma definição em símbolos e linguagem matemática. Note o estilo da definição:

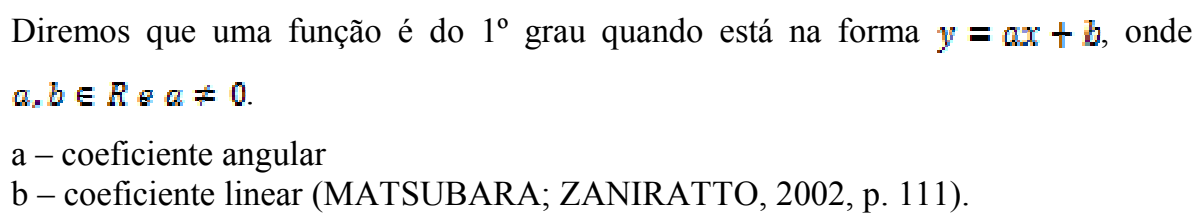

\subsubsection{Livro F}

O livro apresenta aplicações contextualizadas, tabelas, comentários, imagens, exercícios problematizados em maioria, de oito nesta seção, apenas dois não são contextualizados, sugestões de uso da calculadora para compreensão de conceitos, dentre outras estratégias. Existe também uma seção que mescla curiosidade com aprofundar no tema. Bem como no anterior, não aparece nenhuma definição formal para função. Apenas novamente quando se fala em função afim.

Função afim é toda função que pode ser expressa por uma fórmula do tipo $y=a x+b$, com $a \neq 0$. (MORI; ONAGA, 2012, p. 215).

\section{CONSIDERAÇÕES FINAIS}

Conforme indicado no início do presente artigo, a intenção deste não é a de indicar uma forma correta para trabalho com ensino de matemática, nem dizer que uma obra é boa ou ruim, apenas fazer comparações e levar a reflexões. É possível concluir que os momentos históricos influenciam diretamente nas práticas adotadas. Isso porque se percebe que em época de positivismo e foco na necessidade de mão de obra técnica urgente, os livros eram mais diretos e não se preocupavam em contextualizar a matemática com o mundo real. $\mathrm{E}$ atualmente que a situação do Brasil mudou, as abordagens dos livros também. Seguindo a 


\section{\% FORSCIENCE}

CRUZ, A. R. B. da. Influências históricas na abordagem de livros didáticos de matemática

evolução da ciência, os livros buscam evoluir ao ligar a matemática com o mundo real. $\mathrm{Ou}$ seja, acontecimentos políticos ou ampliação tecnológica afetam o contexto apresentado nos livros didáticos acompanhando as mudanças sociais e culturais.

Observando a evolução histórica dos livros didáticos, desde a década de 1970, até a atual, é possível conferir a grande influência de movimentos pautados no positivismo ao início chegando ao final para uma forma de apresentação de conceitos mais contextualizados à realidade e de forma menos voltada ao conteúdo por si só. As abordagens antes com teorias complexas de matemática e exercícios puramente mecânicos deram lugar para abordagens mais próximas da realidade dos alunos, bem como mais reflexivas. Não é possível afirmar que todos os livros atuais estão completamente contextualizados com a realidade do mundo, mas já se percebe que as influências positivistas e da Matemática Moderna, diminuíram muito, tendo em vista que os livros atuais dedicam maior atenção à contextualização.

Nem sempre o que está em voga é o melhor a ser feito, toda via, não se pode ignorar que num mundo com tanta tecnologia como o atual os alunos não são atraídos por uma matemática sem contexto com a realidade ou o próprio mundo da informática. Acredito que o presente trabalho contribuiu para uma reflexão acerca do processo de ensino e aprendizagem de matemática. A pesquisa levou ao pensamento da importância do contexto cultural, pois ele foi e é capaz de afetar os livros didáticos, logo, nós os professores também devemos ser afetados. Além disso, consigo pensar que o planejamento das aulas, deve levar em conta o momento histórico e o contexto cultural, isto porque ambos dizem de sentidos que cabem ser tomados no tratamento de questões matemáticas. A história e a cultura, em outras palavras, constituem a significação de signos lógicos; sabê-las é função do trabalho docente implicado à ideia de educar, ajudar a posição-discente fazer travessias no/pelo saber.

De certo modo, seria complexo o professor atual trabalhar reproduzindo apenas algo parecido com o que era feito na década de 70, onde era presente uma matemática muito formal e sem contextualização com o cotidiano. Até porque seria anacrônico: estar no século XXI e usar metodologias de ensino exclusivamente da década de 1970. Novamente informando, que isso não é errado, porém, pode ser inviável. Talvez seja esta uma razão, aliada à estudos da educação matemática, para os livros atuais terem abordagens mais diversificadas e menos teóricas. 


\title{
HISTORICAL INFLUENCE ON APPROACH OF TEACHING MATHEMATICS BOOKS
}

\begin{abstract}
This article aims to take the reader to a reflection of mathematics teaching practice. In this purpose, historical information from 1970s to the present was sought, the search was done in articles and works for the period, beyond analyzing textbooks of mathematics related to the time slot, it was a total of six textbooks between the years 1971 and 2012. It was consulted authors as Burigo (1990), D'Ambrósio (2008), DobrovolskI (2009), Iskandar (2002), Kline (1976), Mathias (2012) e Valente (2007), to better understand each historical moment of mathematics in Brazil, having the period set by reference. The National Curriculum Parameters (PCNs) of math were also fundamental to justify the strong relationship between science and history. It is expected that at the end of reading it is clear the relationship of mathematics with its history and the history of the country and also that mathematics education is dynamic and tracks the social moment of the country.
\end{abstract}

Keywords: Textbook. Mathematics’ History. Contextualized Approaches.

\section{REFERÊNCIAS}

BRASIL. Secretaria de Educação Fundamental. Parâmetros curriculares nacionais: matemática. Brasília: MEC/SEF, 1997. 142 p.

BURIGO, E. Z. Matemática Moderna: progresso e democracia na visão de educadores brasileiros nos anos 60. Teoria \& Educação, Porto Alegre, n. 2, 1990.

D'AMBROSIO, U. Uma história concisa da matemática no Brasil. Petrópolis, RJ: Vozes, 2008. $126 \mathrm{p}$.

DOBROVOLSKI, E. N.; PINTO, N. B. Movimento da matemática moderna nas práticas escolares e suas repercussões na maneira de ensinar. In: CONGRESSO NACIONAL DE EDUCAÇÃO - EDUCERE, 9., 2009, Curitiba. Anais... Curitiba: Champagnat, 2009. v. 1.

GIOVANNI, J. R.; CASTRUCCI, B. A conquista da matemática: teoria, aplicação, $8^{a}$ série. São Paulo: FTD, 1985. 192 p.

; GIOVANNI JÚNIOR, J. R. Matemática: pensar e descobrir, 8. São Paulo: FTD, 1996. 303 p. (Coleção pensar e descobrir)

ISKANDAR, J.; LEAL, M. R. Sobre o positivismo e educação. Revista Diálogo

Educacional, Curitiba, v. 3, n. 7, p. 89-94, set./dez. 2002.

KLINE, M. O fracasso da matemática moderna. São Paulo: IBRASA, 1976. 211 p. 
MATHIAS, C. E. M. Novas tecnologias no ensino da matemática: repensando práticas. 2. ed. Brasília: Capes/UAB MEC, 2012. Disponível em: <http://ntem.lanteuff.org.>. Acesso em: 22 set. 2015.

MATSUBARA, R.; ZANIRATTO, A. A. Matemática: história, evolução, conscientização, $8^{\mathrm{a}}$ série. 2. ed. São Paulo: IBEP, 2002. 215 p. (Coleção Big Mat).

MORANDI, H. Matemática: método moderno: curso médio: ciclo ginasial. Rio de Janeiro: Paulo de Azevedo Ltda., 1971. 325 p.

Matemática: método moderno. Curso médio: ciclo ginasial. Rio de Janeiro: Paulo de Azevedo,1971. $325 \mathrm{p}$.

MORI, I.; ONAGA, D. S. Matemática: ideias e desafios, $9^{\circ}$ ano. 17. ed. São Paulo: Saraiva, 2012. 320 p.

PINTO, N. B.; FERREIRA, A. C. da C. O movimento paranaense de matemática moderna: o papel do NEDEM. Revista Diálogo Educacional, Programa de pós-graduação da PUC. v. 6, n. 18, maio/ago. 2006.

SANGIORGI, O. Matemática: 8. São Paulo: Nacional, 1978. 198 p.

VALENTE, W. R. A matemática moderna nas escolas do Brasil e de Portugal: história e epistemologia. In MATOS. J. M.; VALENTE. W.R. (Org.). A matemática moderna nas escolas do Brasil e de Portugal: primeiros estudos. São Paulo: Da Vinci, 2007.

Recebido em: 21/09/2015

Aprovado em: 20/02/2016

Publicado em: 05/07/2016 